\title{
Grippeimpfung für Gesundheitsberufe
}

\section{Geschützte Gesundheitsmitarbeiter schützen auch Patienten und Pflegeheimbewohner}

Schwere Grippewellen können pro Jahr bis zu 840.000 Menschen in Österreich betreffen. Auch wenn Derartiges derzeit nicht absehbar ist, sind immungeschwächte Personen, wie ältere Menschen, kleine Kinder und chronisch Kranke auch bei leichten Grippewellen einem erhöhten Gesundheitsrisiko ausgesetzt. Pro Jahr sterben in Österreich etwa 1000 Personen an den Folgen einer Grippeinfektion.

Das Risiko an einer Influenza zu erkranken, kann durch eine Impfung beträchtlich gesenkt werden - vor allem in der Altersgruppe von 18 bis 65 Jahren. Auch wenn die Wirkung der Influenza-Impfung in der Altersgruppe der über 65-Jährigen reduziert ist, bietet sie eine hohe Schutzwirkung. Sie kann mehr als die Hälfte in dieser Altersgruppe vor einer Erkrankung und ebenso große Anteile vor der Hospitalisierung und vor dem Tod bewahren.

Die Durchimpfungsraten in Österreich sind sehr niedrig, stellt Univ.-Prof. Dr. Ursula Wiedermann-Schmidt, Leiterin des Instituts für Spezifische Prophylaxe und Tropenmedizin an der Medizinischen Universität Wien fest. In der Gruppe der ältesten Personen liegt sie weit unter 20 Prozent. Ebenso niedrig ist die Durchimpfungsrate bei Personen, die im Gesundheitswesen tätig sind (HCW) mit nur etwa

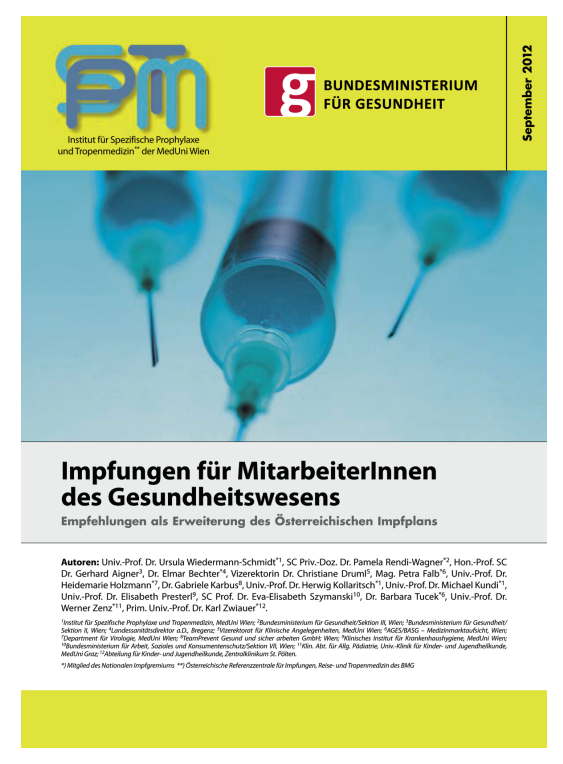

17 Prozent. Gründe für eine geringe Bereitschaft sich impfen zu lassen liegen in der Angst vor Nebenwirkungen aber auch in der Annahme, dass die Impfung nicht protektiv wirksam sei. Gerade im Bereich der Krankenversorgung und -pflege (besonders im geriatrischen Bereich) konnte aber in Studien sehr eindrucksvoll gezeigt werden, dass durch Impfung des Pflege/ Ärztepersonals die Erkrankung und Sterbefälle bei den kranken Personen signifikant reduziert werden konnte. Das Risiko der Erkrankung und der Weitergabe der Infektion durch HCW ist, so die neuen österreichischen Empfehlungen für Gesundheitspersonal, sehr hoch. Etwa 23 Prozent aller Mitarbeiter im Gesundheitssystem machen in einer milden Saison eine Serokonversion durch, wobei ein Drittel aller Erkrankungen subklinisch verläuft. Gesundheitspersonal kann daher für eine Transmission große Bedeutung haben.

Besonders empfohlen wird die Impfung für alle Personen mit erhöhter Gefährdung infolge eines Grundleidens, Schwangere und Frauen, die während der Influenzasaison schwanger werden wollen, Kinder ab dem 7. Lebensmonat bis 4 Jahre, Personen über 50 Jahre und insbesondere ab 65 Jahren, stark übergewichtige Personen, Betreuungspersonen und Haushaltskontakte von Risikogruppen, Personen aus Gesundheitsberufen und Personen mit häufigem Publikumskontakt.

\section{Erweiterung des Impfplans für Gesundheitsmitarbeiter}

Aufgrund der generell niedrigen Durchimpfungsraten, nicht nur die Influenzaimpfung betreffend, des Gesundheitspersonals, hat das Nationale Impfgremium mit erweiterter Expertenrunde und in Kooperation mit dem Gesundheitsministerium nun ein ergänzendes Schriftstück zum Österreichischen Impfplan bezüglich Impfungen für Gesundheitspersonal herausgegeben, berichtet Wiedermann. Ziel dieser Empfehlungen ist, die nötigen Informationen zu den empfohlenen Impfungen sowie entsprechende Hilfestellung in der Umsetzung dieser anbieten zu können.

Der Österreichische Impfplan empfiehlt für Gesundheitspersonal (HCW) grundsätzlich die gleichen Basis-Impfun-

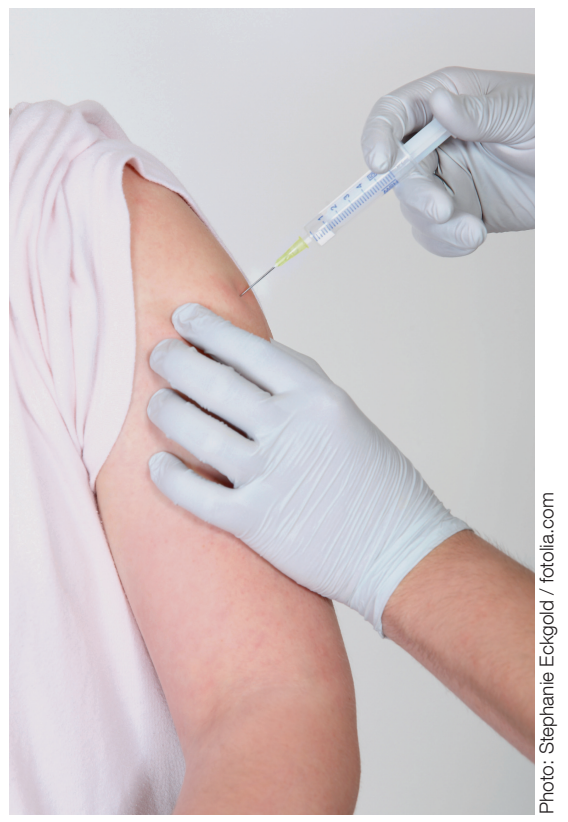

gen wie für alle anderen Erwachsenen. Dies umfasst, so die aktuellen Empfehlungen als Erweiterung des Österreichischen Impfplans zu Impfungen für Mitarbeiter des Gesundheitswesens, zunächst den routinemäßigen Schutz vor Diphtherie, Tetanus, Pertussis, Polio, Masern, Mumps, Röteln und Varizellen. „Für jegliche HCW wichtig ist darüber hinaus der Schutz vor Hepatitis A und B sowie die jährliche Influenzaimpfung. Ein zusätzlicher Schutz vor Meningokokken (tetravalenter Konjugatimpfstoff) wird laut dem Österreichischen Impfplan bei besonderer Exposition an Versorgungseinrichtungen für Pädiatrie und/oder Infektionskrankheiten und für Laborpersonal empfohlen."

Die WHO-Empfehlung für die Zusammenstellung der Influenzaimpfstoffe für die nördliche Hemisphäre in der Saison 2012/2013 beinhaltet H1N1: A/California/7/2009, H3N2: A/Victoria/361/2011 und B: Wisconsin/1/2010. Der beste Zeitpunkt für die Impfung ist zwischen Oktober und Dezember. Auch während der Grippewelle ist die Impfung noch sinnvoll, solange noch keine Ansteckung erfolgt ist bzw. noch keine Krankheitszeichen auftreten.

Quelle: Journalistenseminar

„Influenza - Ein Gesundheitsrisiko?" 